\title{
The Hard X-Ray Emission from Scorpius X-1 as Seen by INTEGRAL
}

\author{
Steven J. Sturner*1,2 and Chris R. Shrader ${ }^{1,3}$ \\ ${ }^{1}$ CRESST and Astroparticle Physics Laboratory, NASA/GSFC, Greenbelt, MD 20771, USA \\ ${ }^{2}$ Center for Space Science and Technology, University of Maryland, Baltimore County, Baltimore, \\ MD 21250, USA \\ ${ }^{3}$ Universities Space Research Association, Columbia, MD 21044, USA \\ E-mail: steven.j.sturner@nasa.gov, chris.r.shrader@nasa.gov
}

\begin{abstract}
We present the results of our hard X-ray and gamma-ray study of the LMXB Sco X-1 utilizing INTEGRAL data as well as contemporaneous RXTE PCA data. We have investigated the hard $\mathrm{X}$-ray spectral properties of Sco X-1 including the nature of the high-energy, nonthermal component and its possible correlations with the location of the source on the soft X-ray color-color diagram. We find that Sco X-1 follows two distinct spectral tracks when the $20-60 \mathrm{keV}$ count rate is $>130$ counts/second. One state is a hard state which exhibits a significant high-energy, powerlaw tail to the lower energy thermal spectrum. The other state shows a much less significant high-energy component. We found suggestive evidence for a correlation of these hard and soft high-energy states with the position of Sco X-1 on the low-energy X-ray color-color diagram. We have searched for similar behavior in 2 other Z sources: GX 17+2 and GX 5-1 with negative results.
\end{abstract}

7th INTEGRAL Workshop

September 8-11 2008

Copenhagen, Denmark

* Speaker. 


\section{Introduction}

Weakly magnetized neutron star Low-Mass X-ray Binaries (LMXBs) display complex X-ray spectra that are not well understood. Nonetheless, classifications of LMXB spectra based on their observed behavior in the X-ray color-color diagrams (CCDs) and their correlated temporal properties provide a reasonably coherent empirical picture in the 2-10-keV domain (e.g. [1]). This leads, for example, to the broad classifications of $\mathrm{Z}$ sources (with luminosities near the Eddington luminosity, $L_{e d d}$ ) and atoll sources (with luminosities of $\sim 0.01-0.1 L_{e d d}$ ). Convincing evidence supports the idea that the mass accretion rate in the $\mathrm{Z}$ sources increases from the top left to the bottom right of the $\mathrm{Z}$ pattern, i.e. along the so-called horizontal (HB), normal (NB), and flaring branches (FB). Each of these branches is well defined in terms of its temporal characteristics.

Atoll source spectra are often found to extend up to energies $>50 \mathrm{keV}$, analogous perhaps to the hard X-ray component of black hole binaries. On the other hand, Z sources have comparatively soft spectra, dominated by thermal or Comptonized thermal components. Hard X-ray emission above $20 \mathrm{keV}$ has been detected from Z sources, notably Sco X-1 (e.g. [2, 3]), GX 5-1 [4], GX 17+2 [5], GX 340+0 [6], and GX 349+2 [7]. Cir X-1 is sometimes characterized as a Z source and has been detected above $50 \mathrm{keV}$ [8]. Cyg X-2 is not (or is only marginally) detected above $30 \mathrm{keV}$ [9]. Generally this hard emission is of a transient or variable nature and its characteristics are yet to be clearly defined.

Precise spectral modeling, even within the soft band, is still a subject of debate, and it is not clear exactly how the proposed model components may evolve to form the $\mathrm{Z}$ track, or lead to the underlying synergy between the $\mathrm{Z}$ and atoll patterns. The relationship between the hard ( $\gtrsim 20 \mathrm{keV})$ $\mathrm{X}$-ray behavior as a function of the source color-color ( $\mathrm{Z}$ or atoll track) configuration has proven similarly elusive, although studies to date are much less comprehensive. The hard X-rays thus may hold important undiscovered clues. Supporting this assertion, recent work strongly suggests that the predominant variable component in these systems is associated with Comptonized thermal emission associated with the boundary layer $[10,11]$. Since Comptonization is the likely source of the hard-X-ray emission components seen with INTEGRAL, it is reasonable to anticipate correlated behavior. It is thus pertinent to search for some coherent empirical picture regarding the hard X-ray CCD patterns.

In this paper we report on our study of the hard X-ray spectral properties of Sco X-1 using data from the IBIS/ISGRI imager on INTEGRAL and the PCA on RXTE. In this study our goal was to look for correlations between the hard and soft X-ray spectral properties of this source. We did this by producing a hard X-ray hardness-intensity diagram (HID) for Sco X-1 using ISGRI data and then looking for correlations between the position of Sco X-1 on that diagram with the temporally coincident position on the soft X-ray CCD. We also performed spectral analysis of Sco X-1 using the ISGRI data for various states associated with regions on the HID. We then produced HIDs for the Z sources GX 5-1 and GX 17+2 to look for behavior similar to that found in Sco X-1.

\section{Observations and Data Analysis}

As previously stated, we used data from the INTEGRAL/IBIS imager for this study. IBIS is a coded mask instrument with a $9^{\circ} \times 9^{\circ}$ fully coded field of view and a 12' (FWHM) point spread 
function. It is sensitive over the energy range $15 \mathrm{keV}$ to $10 \mathrm{MeV}$. There are two detector layers: ISGRI, an upper CdTe layer with peak sensitivity between 15 and $200 \mathrm{keV}$, and PICsIT, a bottom CsI layer, with a peak sensitivity above $200 \mathrm{keV}$. Here we have used only ISGRI data. The data for Sco X-1 consisted of 960 science windows (SCWs) ranging from March 28, 2003 to August 25, 2006 with a total exposure of $1.77 \mathrm{Msec}$. This dataset is much larger than the one previously used by Di Salvo et al. (2006) [14]. The data used for GX 17+2 and GX 5-1 analyses consisted of 1732 SCWs ranging from April 17, 2003 to April 22, 2007 with a total exposure of $2.62 \mathrm{Msec}$.

INTEGRAL data reduction was performed using the standard OSA 7 analysis software package available from the INTEGRAL Science Data Centre. Spectral analysis was performed using the XSPEC data analysis package, and timing analysis was performed using the XRONOS data analysis package. We searched the RXTE/PCA public data archive at the HEASARC for observations that were concurrent with the INTEGRAL observations. We analyzed the PCA data from these observations using FTOOLS suite of software available from the HEASARC.

\section{Results - Sco X-1}

\subsection{Hardness-Intensity Diagram}

Using these INTEGRAL observations, we performed a multi-epoch lightcurve analysis leading to the HID presented in Figure 1. This HID illustrates the presence of distinct hard and soft spectral states when the $20-60 \mathrm{keV}$ count rate is $\gtrsim 130 \mathrm{cts} / \mathrm{s}$, forming a V-shaped locus of points with the apex towards the lower intensity side of the diagram. We note that, interestingly, soft-band

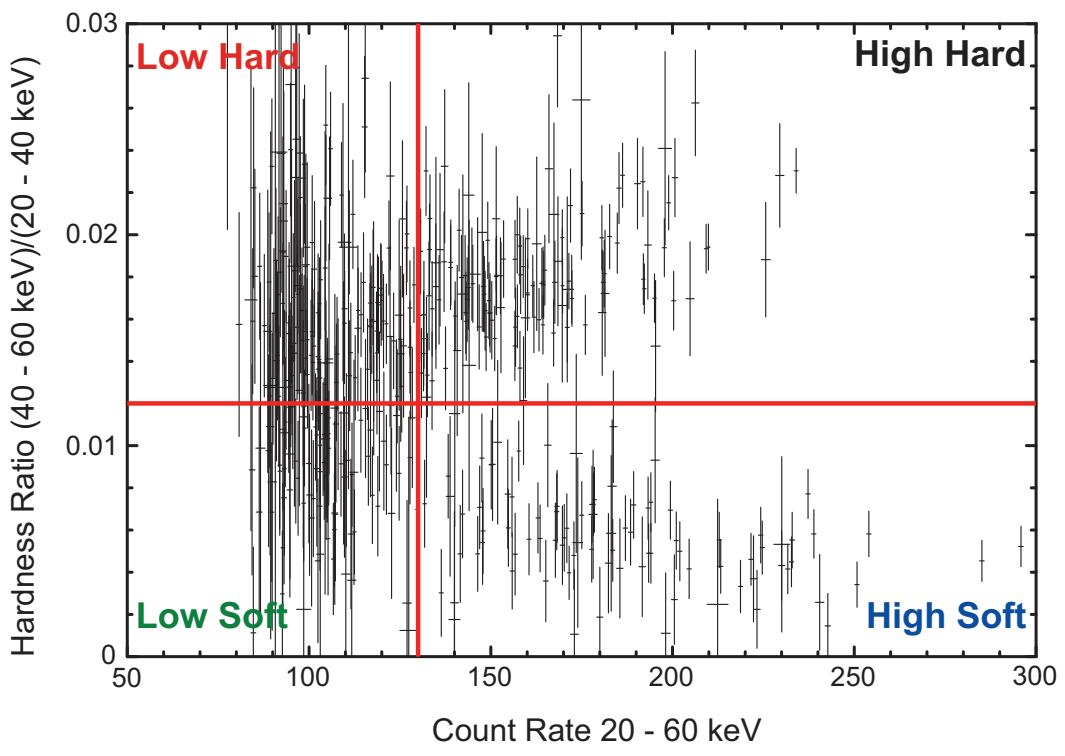

Figure 1: Hardness-Intensity diagram (HID) for Sco X-1 from IBIS/ISGRI data. Each data point represents 2 hours of data. The diagram shows distinct hard and soft spectral states. 
HIDs for both GX 5-1 [4] and GX 17+2 [12] exhibit a V-shaped locus as well, however with the opposite sense; the apex is on the high intensity side of the diagram. In these cases the HIDs were constructed using INTEGRAL/JEM-X data in the the 3-5 keV and 5-12 keV bands for GX 5-1 and BeppoSAX data in the the 4.5-7 keV and 7-10.5 keV bands for GX $17+2$.

\subsection{Spectral States}

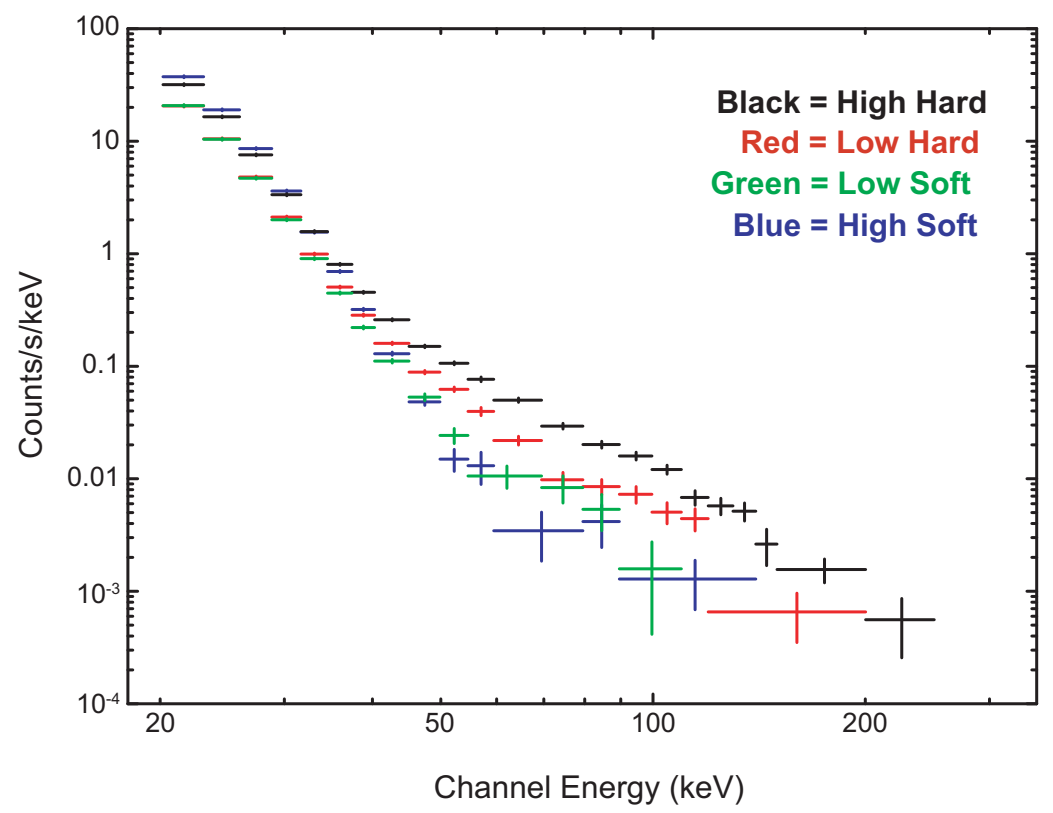

Figure 2: Average IBIS/ISGRI count spectra for the 4 states labeled in Figure 1. Note the two distinct states below $\sim 35 \mathrm{keV}$ and the large variations in the spectra above $40 \mathrm{keV}$.

In Figure 1 we have divided the HID into 4 quadrants: high hard, high soft, low hard, and low soft. We have performed spectral analysis of Sco X-1 using the ISGRI data for time periods when the source was in each of those four states. We produced average spectra for each state. This was done by associating the time periods pertaining to each point in a given quadrant of Figure 1 to a $\mathrm{SCW}$. The resulting average spectra are shown in Figure 2. It is readily seen that the high hard state exhibits a significant hard X-ray tail that extends past $200 \mathrm{keV}$. On the the other hand the high soft state shows much weaker emission above $40 \mathrm{keV}$. We have fit these spectra with a model consisting of thermal bremsstrahlung and power law components. We find that the temperature of the thermal component in the four states ranges from $4.31 \mathrm{keV}$ in the high soft state to $4.48 \mathrm{keV}$ in the high hard state with typical errors of $\sim 0.1 \mathrm{keV}$. The best fit power law index is $2.48 \pm 0.11$ for the high hard state and $3.12 \pm 0.25$ for the low hard state.

\subsection{Correlation Between the HID and the CCD}

Over the years, there have been multiple reports of both detections and non-detections of a hard nonthermal component to the X-ray spectrum of Sco X-1 and efforts to correlate its intensity 


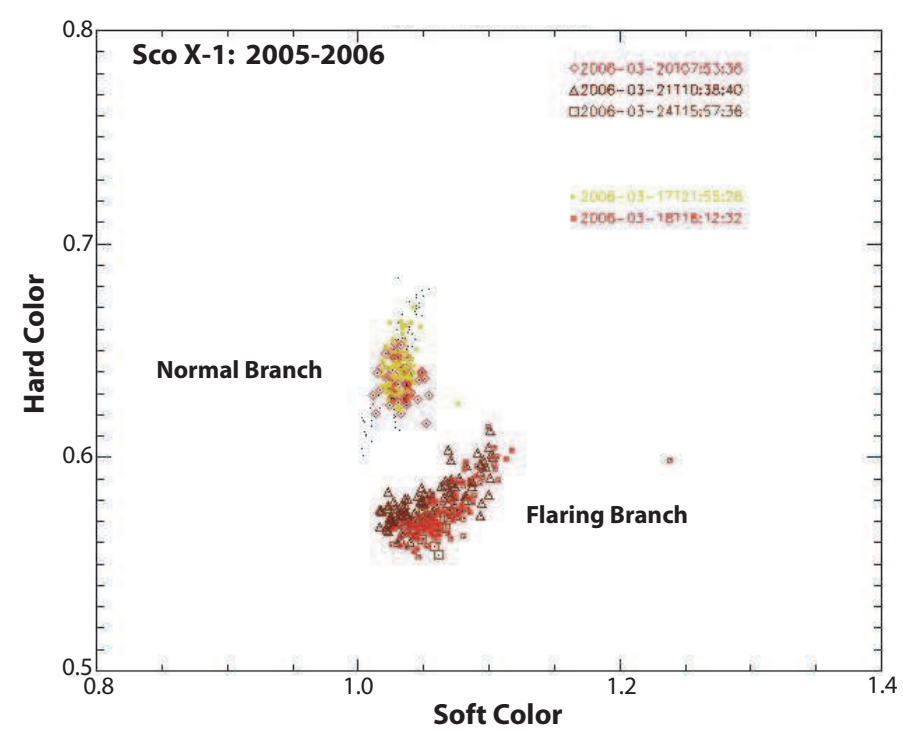

Figure 3: A Sco X-1 X-ray color-color diagram (CCD) derrived from RXTE/PCA data. The data were chosen to due to there temporal overlaps with the INTEGRAL observations.

with positions on the soft X-ray CCD. Recently, it has been shown that this hard component is variable but efforts to associate it with particular states corresponding to certain positions on the soft X-ray CCD have met with mixed success. Strickman \& Barret (2000) [13] found evidence using CGRO/OSSE and RXTE/PCA that the hard component was most prevalent when Sco X-1 was positioned near the junction of the NB and FB on the CCD. On the other hand, D'Amico et al. (2001) [2] found using both HEXTE and PCA on RXTE that there was no correlation between the presence of the hard tail and the position on the CCD but the hardest power-law indices where found when the source was on the FB. In contrast, Di Salvo et al. (2006) [14] found using INTEGRAL/ISGRI and RXTE/PCA that the flux in the hard component decreased as the source moved in the $\mathrm{CCD}$ in the sense of increasing mass accretion rate, i.e. $\mathrm{HB} \rightarrow \mathrm{NB} \rightarrow \mathrm{FB}$.

We have constructed our own CCD for Sco X-1 using archival RXTE/PCA data coincident with our INTEGRAL data. We chose the four energy bands: $2.0-3.5 \mathrm{keV}, 3.5-6.0 \mathrm{keV}, 6.0-9.7 \mathrm{keV}$, and 9.7-16.0 keV. The result is shown in Figure 3. To look for a correlation between the four states shown in the hard X-ray HID (Figure 1) and the temporally coincident positions on the CCD in Figure 3, we filtered the light curves used to produce Figure 1 excluding all data not within \pm 6 hours of the times listed on Figure 3. Replotting Figure 1 using only temporally coincident data yields Figure 4. We have color coded the data to highlight data taken when Sco X-1 was in the NB and FB portions of the CCD. This analysis shows a correlation between the soft X-ray CCD and the hard X-ray HID. Figure 4 indicates that when Sco X-1 is on the normal branch of the CCD it is preferentially in the hard state and, conversely, it is preferentially in the soft state when on the FB of the CCD.

\section{Results - GX 17+2 \& GX 5-1}

We have constructed INTEGRAL HIDs similar to Figure 1 for GX 17+2 (Figure 5) and GX 


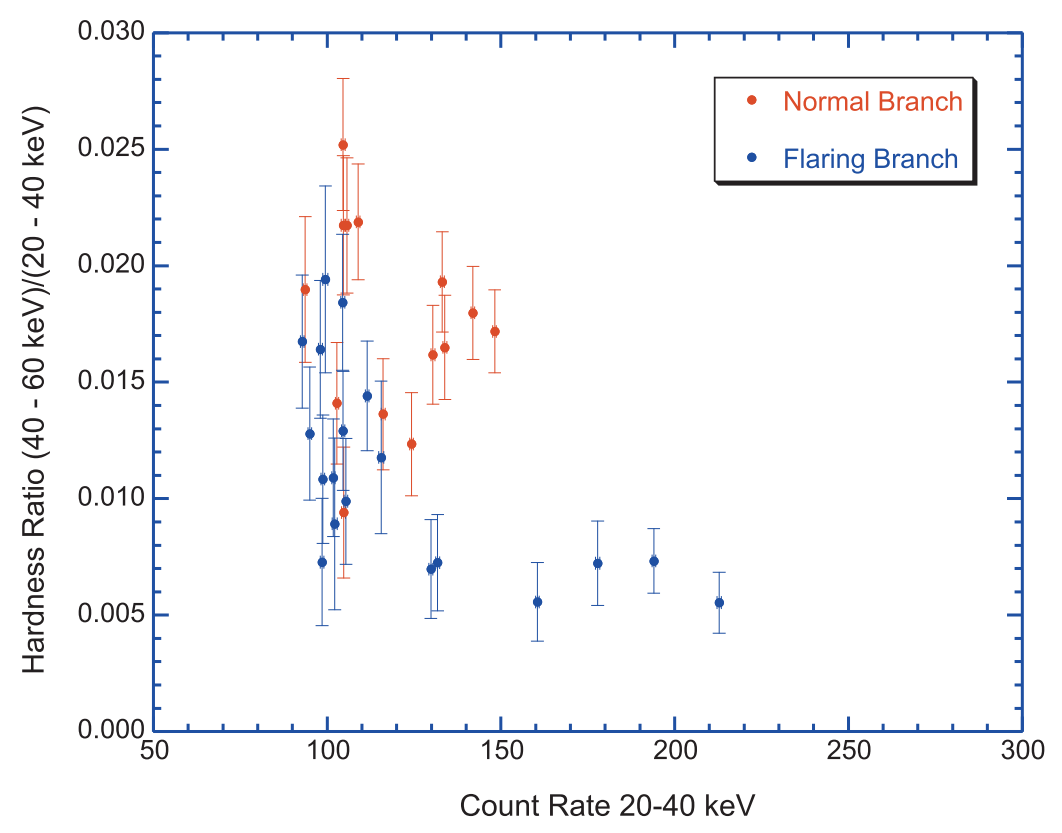

Figure 4: HID for Sco X-1 using the subset of the IBIS/ISGRI data in Figure 1 which fell within \pm 6 hours of a RXTE/PCA observation shown in Figure 3.

5-1 (Figure 6). In these cases, there are no well defined tracks. Also, the hardness ratio (defined the same as in our Sco X-1 analysis) extends to higher values, i.e. these are overall harder sources. Thus both GX 17+2 and GX 5-1 exhibit distinctly different HID behavior than Sco X-1. As noted earlier, it is of interest that both GX 17+2 and GX 5-1 have been shown to exhibit a V-shape structure to their soft X-ray HIDS using data in the range 3-12 keV but that this "V" is oriented in the opposite sense with the vertex towards higher intensities $[4,12]$.

\section{Discussion}

It appears from this analysis that Sco $\mathrm{X}-1$ is a unique case, in that two distinct hard and soft tracks are evident in the hard X-ray HID. Not only do GX 5-1 and GX 17+2 sources lack this behavior, there is no distinct emergent morphology. Clearly, Sco X-1 appears unique among the Z-sources that are routinely detected above $40 \mathrm{keV}$. Why should this be the case? If the hard emission is due to Comptonization this suggests some population of energetic electrons which appears intermittently. If this hard emission is associated with the Normal-branch of the Z-track, it could be the case that Compton cooling effects suppress it in the Flaring branch. One possible explanation is that Sco X-1 has a synchrotron component accounting for or contributing to its hard $\mathrm{X}$-ray spectrum. Indeed its known to be a radio source - typically $\sim 5 \mathrm{mJy} @ \sim 1 \mathrm{Ghz}$, and it exhibits a hot-spot structure on $\sim 10$ mas scales reminiscent of radio Galaxy jet - lobe morphologies [15]. We also note that Shabazz et al. (2008) [16] find IR (2.4 micron) polarization, further supporting the synchrotron hypothesis. Coordinated radio - hard X-ray observations could help clarify this. 


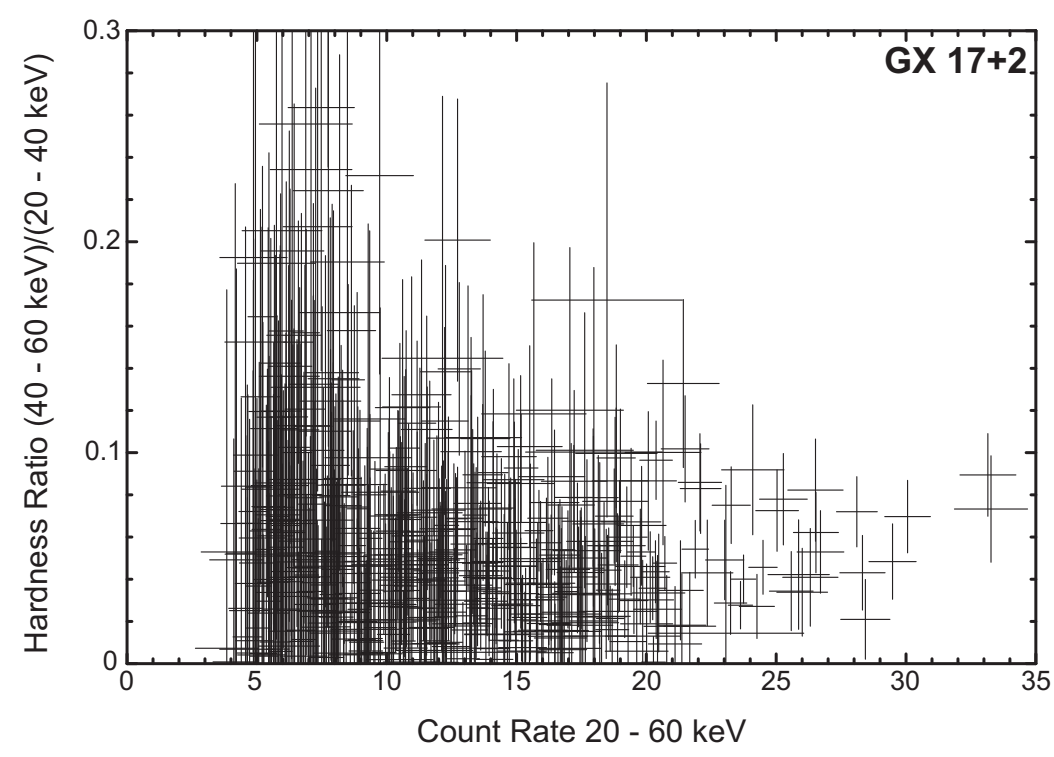

Figure 5: HID for GX 17+2 from IBIS/ISGRI data. Each data point represents 2 hours of data.

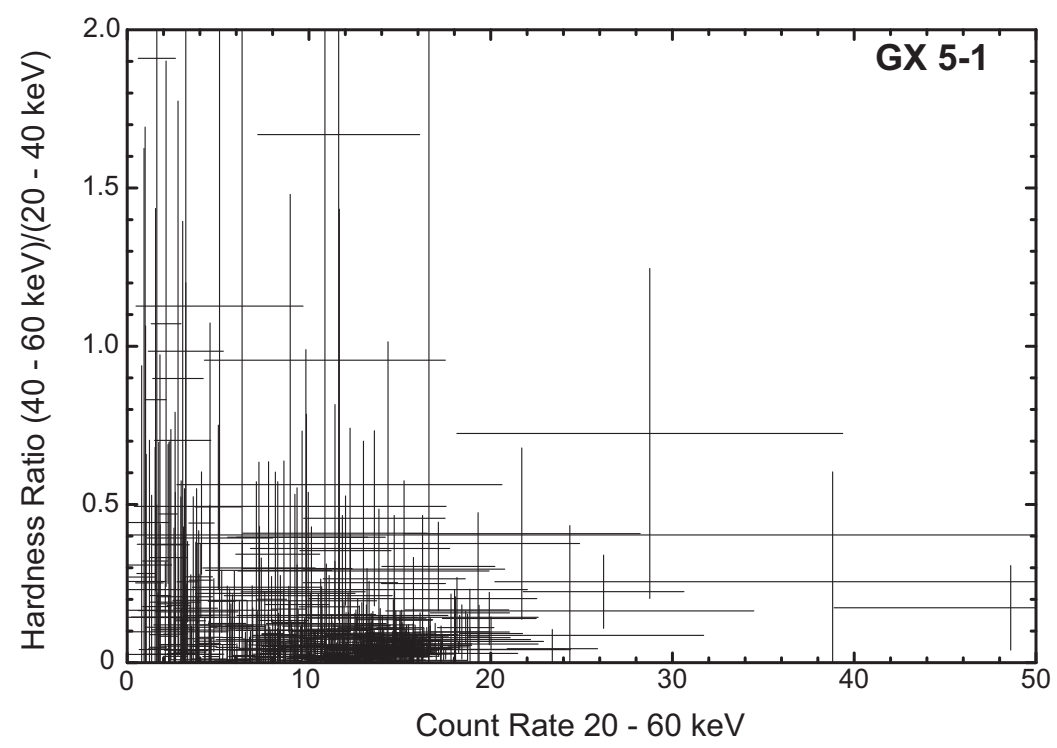

Figure 6: HID for GX 5-1 from IBIS/ISGRI data. Each data point represents 2 hours of data. 


\section{References}

[1] M. van der Klis, A Review of Rapid X-ray Variability in X-ray Binaries, in Compact Stellar X-Ray Sources, eds. W. Lewin \& M. van der Klis, Cambridge University Press, 39, 2004 [astro-ph/0410551]

[2] F. D'Amico, W. A. Heindl, R. E. Rothschild, \& D. E. Gruber,High-Energy X-Ray Timing Experiment Detections of Hard X-Ray Tails in Scorpius X-1, ApJ 547 (L147) 2001

[3] S. J. Sturner, C. R. Shrader, \& G. Weidenspointner, The Hard X-Ray Emission from Scorpius X-1 as Seen by INTEGRAL, BAAS 40 (46) 2008

[4] A. Paizis, et al., Resolving the Hard X-Ray Emission of GX 5-1 with INTEGRAL, A\&A 433 (599) 2005

[5] R. Farinelli, L. Titarchuk, \& F. Frontera, The Hard X-Ray Tails in Neutron Star Low-Mass X-Ray Binaries: BeppoSAX Observations and Possible Theoretical Explanation of the Case of $G X 17+2$, ApJ 662 (1167) 2007

[6] G. Lavagetto, et al., A BeppoSAX Study of the Galactic Z-Source GX 340+0, NuPhysB 132 (616) 2004

[7] T. Di Salvo, et al., Detection of a Hard Tail in the X-Ray Spectrum of the Z Source GX 349+2, ApJ 554 (49) 2001

[8] R. Iaria, T. Di Salvo, N. R. Robba, \& L. Burderi, Study of the Circinus X-1 Broadband Spectrum at Orbital Phases Close to the Apoastron, ApJ 567 (503) 2002

[9] G. Lavagetto, et al., The Broad-Band Spectrum of Cyg X-2 with INTEGRAL, A\&A 445 (1089) 2006

[10] M. G. Revnivtsev, \& M. R. Gilfanov, Boundary Layer Emission and Z-Track in the Color-Color Diagram of Luminous LMXBs, A\&A 453 (253) 2006

[11] C. R. Shrader, P. Reig, \& D. Kazanas, Fourier-Resolved Spectroscopy of 4U 1728-34: New Insights into Spectral and temporal Properties of Low-Mass X-Ray Binaries, ApJ 667 (1063) 2007

[12] T. Di Salvo, et al., The Discovery of a State-Dependent Hard tail in the X-ray Spectrum of the Luminous Z Source GX 17+2, ApJ 544 (L119) 2000

[13] M. Strickman, \& D. Barret, Detection of Multiple Hard X-Ray Flares from Sco X-1 with OSSE, in proceedings of The Fifth Compton Symposium, ed. M. L. McConnell, \& J. M. Ryan, AIP, 222, 2000

[14] T. Di Salvo, et al., A Hard X-Ray View of Scorpius X-1 with INTEGRAL: Nonthermal Emission?, ApJ 649 (L91) 2006

[15] E. B. Fomalont, B. J. Geldzahler, \& C. F. Bradshaw, Scorpius X-1: The Evolution and Nature of the Twin Compact Radio Lobes, ApJ 558 (283) 2001

[16] T. Shahbaz, R. P. Fender, C. A. Watson, \& K. O’Brien, The First Polarimetric Signatures of Infrared Jets in X-Ray Binaries, ApJ 672 (510) 2008 